DOI: $10.17957 / \mathrm{IJAB} / 15.1788$

http://www.fspublishers.org

\title{
Virus-induced Silencing SBEIIa and SSIIa, Alone or Together, Increased the Amylose and Resistant Starch Contents in Spring Wheat
}

\author{
Zhaofeng $\mathrm{Li}^{1}$, Weihua $\mathrm{Li}^{1}$, Wei Liu ${ }^{2}$, Fubo Nan ${ }^{1}$ and Donghai Zhang ${ }^{*}$ \\ ${ }^{1}$ College of Agriculture, Shihezi University, Shihezi, Xinjiang 832000, P. R. China \\ ${ }^{2}$ College of Medicine, Shihezi University, Shihezi, Xinjiang 832000, P. R. China \\ *For correspondence: donghaizhng@163.com \\ Received 19 October, 2020; Accepted 08 March 2021; Published 10 May 2021
}

\begin{abstract}
Foods rich in amylose and resistant starch (RS) have great potential to improve human health and lower the risk of noninfectious diseases. Common wheat (Triticum aestivum L.) is a major staple food crop with low RS content in the grains. The content of RS, preferentially derived from amylose, may be increased by suppressing amylopectin synthesis via silencing the starch branching enzyme (SBE) II a or/and starch synthase (SS) II a. In this study, SBEIIa and SSIIa were silenced separately and simultaneously using a barley stripe mosaic virus-virus-induced gene silencing (BSMV-VIGS) system. Compared with grains from control BSMV:00-inoculated spikes, grains from BSMV:SBEIIa- and BSMV:SSII a-infected spikes had fewer SBEIIa and SSIIa transcripts, together with increased amylose contents (18.62 and 24.48\%, respectively) and RS contents (11.61 and 16.67\%, respectively). Infection with BSMV:SBEIIa-SSII a reduced SBEII a and SSII a transcript levels and increased the amylose and RS contents (32.02 and 22.33\%, respectively). Thus, BSMV-VIGS is a useful tool for the rapid silencing of single or multiple starch synthase-related genes and BSMV shows great potential to study the functions of genes involved in starch biosynthesis or other processes/traits in developing grains. We showed that the SSII $a$ gene plays an important role in the synthesis of amylose and RS and that the effects of simultaneously silencing SBEII $a$ and SSIIa on starch synthesis are greater than those of single gene silencing. Our study lays the foundation for the molecular design-based breeding of high-amylose and high-RS wheat. (C) 2021 Friends Science Publishers
\end{abstract}

Keywords: BSMV-VIGS; Amylose; Resistant starch; Wheat

\section{Introduction}

Starch is the most consumed carbohydrate in the human diet. Although most of the starch is degraded by specific enzymes and absorbed in the small intestine, a fraction of the starch, known as resistant starch (RS), transits through the small intestine under undigested state. Upon entering the large intestine, this RS is fermented by the microflora to produce short chain fatty acids (Topping and Clifton 2001). RS exerts the same or a similar function as dietary fiber and has been recognized as a beneficial carbohydrate by the World Health Organization (Asp et al. 1993). Several recent studies have indicated that RS might play a protective role against numerous diseases, including colon cancer (Hylla et al. 1998), colorectal neoplasia (Young and Leu 2004), diabetes (Kim et al. 2003), obesity (Zhou et al. 2009), and inflammatory bowel disease (Moreau et al. 2003). Evidence also indicates that RS enhances the absorption of many minerals (Lopez et al. 2001). These results suggest that increasing RS levels in the diet may be beneficial for human health.

Mutations in genes encoding key enzymes involved in starch biosynthesis may alter the amylose and RS contents in cereal grains. For example, mutation of the amylose extender in maize increases amylose content by $80 \%$. This highamylose maize may be added to increase the amount of RS in wheat products (Brown 2004). In wheat grains, the amylose and RS contents are also increased by knocking out the starch branching enzyme SBEIIa/SBEIIb genes (Regina et al. 2006) or edited SBEIIa gene (Li et al. 2020). Amylose content is also markedly increased in transgenic wheat lines by silencing the SBEIIa gene (Sestili et al. 2010; Hazard et al. 2012). In contrast, silencing the granule-bound starch synthase gene reduces the starch content in wheat (Bennypaul et al. 2012). A mutant wheat line lacking starch synthase (SS)IIa function results in increased amylose content (approximately 35\%) over the wild type line (Yamamori et al. 2000). In barley, the starch structure is altered by the loss of SSIIa, which increases the amylose content (Morell et al. 2003). Loss of SSIIa also affects the starch structure in rice (Umemoto et al. 2002). In maize, SSIIa deficiency alters the structure of amylopectin, which subsequently changes the physicochemical properties of starch (Zhang et al. 2004). Therefore, introducing gene mutations through genetic engineering may be a useful strategy for increasing the amylose and RS contents in human diets. 
Virus-induced gene silencing (VIGS) is a rapid, efficient tool for analyzing gene function in plants (Ratcliff et al. 1997; Baulcombe 1999) that is based on plant RNA interference defense mechanism against viruses. In VIGS, a fragment of a target gene cloned in a viral vector enters the host cell via infection with Agrobacterium or a virus and is converted to long double-stranded RNA (dsRNA) by the RNA-dependent RNA polymerase. In the cytoplasm, these long dsRNAs are then cleaved by the RNase III family enzyme Dicer to yield short interfering RNAs (siRNAs; 2125 bp) with 5' phosphates and 3' dinucleotide overhangs. These siRNAs are then loaded into an RNA-induced silencing complex (RISC) that very efficiently searches the transcriptome for target sequences. When an mRNA target sequence is recognized, the RISC cleaves it. Therefore, VIGS is a type of post-transcriptional gene silencing (Baulcombe 1999; Waterhouse et al. 2001; Rana 2007). VIGS systems have been widely used in dicotyledonous plant species, including tomato (Cox et al. 2019; Bao et al. 2020), tobacco (Tang et al. 2020), and Arabidopsis (CalvoBaltanás et al. 2020). More recently, VIGS has been used in monocotyledonous species, including wheat (Yang et al. 2020), barley (Gunupuru et al. 2019), maize (Murphree et al. 2020), and rice (Purkayastha et al. 2010). Barley stripe mosaic virus (BSMV)-based VIGS has been developed for gene silencing in wheat, and has been reported for silencing genes related to growth and development (Gunupuru et al. 2019; Murphree et al. 2020), resistance to disease and insects (Eck et al. 2010; Yousaf et al. 2013; Yang et al. 2020), and resistance to drought (Kuzuoglu-Ozturk et al. 2012). The BSMV-VIGS system has also been used to investigate the functions of genes affecting wheat grain quality, especially starch and protein biosynthesis (Ma et al. 2012). However, directly improving RS content in spring wheat through manipulating key genes involved in starch biosynthesis by VIGS has not been documented yet.

Wheat is an important cereal crop and one of the sources of nourishment and energy for humankind (Yan et al. 2018). Increasing RS content in wheat is useful for better health of the global population. We envisioned that silencing SBEIIa and SSIIa genes through VIGS might be an alternative way to modify the starch composition of wheat to increase its RS content for human health benefits. In the present study, we separately and simultaneously silenced the SBEIIa and SSIIa genes to investigate the effect of silencing single and multiple genes on the biosynthesis of amylose and $\mathrm{RS}$ in spring wheat through BSMV-VIGS. Our results provide fundamental information for improving $\mathrm{RS}$ content in wheat as well as other cereal crops for global population health benefits.

\section{Materials and Methods}

\section{Plant materials and growing conditions}

Spring wheat (Triticum aestivum L. cv. Xinchun 11) seedlings were grown in a greenhouse at $22-25^{\circ} \mathrm{C}$ and 50 $70 \%$ relative humidity under a 16/8-h light/dark cycle. Wheat spikes at the heading stage were inoculated with BSMV:PDS, BSMV:SBEIIa, BSMV:SSIIa, or BSMV:SBEIIa.-SSIIa. For each treatment, three biological replicates with ten plants per replicate were used. The contents of amylose and RS were determined after the seeds matured.

\section{RNA isolation and cDNA synthesis}

To quantify PDS, SBEIIa, or SSIIa transcription, inoculated spikes were pooled for each of the three biological replicates. At $12 \mathrm{~d}$ after flowering, the embryos were removed from the wheat grains and immediately frozen in liquid nitrogen on harvest. Total RNA was extracted using the TRIzol reagent (TaKaRa Bio, Dalian, China) according to the manufacturer's instructions and then treated with RNase-free DNase I. First-strand cDNA was synthesized using M-MLV reverse transcriptase (TaKaRa Bio).

\section{Construction of BSMV-derived vectors}

BSMV $\alpha, \beta \Delta \beta$, and $\gamma$ plasmids were used as previously described by Holzberg et al. (2002). Four recombinant $\gamma$ vectors, $\gamma: P D S, \gamma: S B E I I a, \gamma: S S I I a$, and $\gamma: S B E I I a-S S I I a$, were constructed to silence target genes (Fig. 1, 2). RT-PCR was used to amplify a 185-bp PDS fragment (FJ517553.1), 178bp SBEIIa fragment (AF286319.1), 171-bp SSIIa fragment (AF155217.2), and 349-bp SBEIIa-SSIIa fragment (including a 178-bp fragment of SBEIIa (AF286319.1), and a 171-bp SSIIa fragment (AF155217.2) using the following specifically designed primers:

PDS-F: 5'-ATATTAATTAACTGGATGAAAAAGCAGGGTGTTCC-3', PDS-R: 5'-TTATGCGGCCGCCTACTTTCAGGAGGATTACCATCC-3', SBEIIa-F: 5'-ATATTAATTAAGACTTGGCAAGTCCGGCGCAACCT-3', SBEIIa-R: 5'-TATGCGGCCGCCGACTAGTTCCTTAACTCCTTTGG-3', SSIIa-F: 5'-ATATTAATTAAAGCCGCTCCAGCCCCGCATGCGTG-3', SSIIa-R: 5'-TATGCGGCCGCTCTGCTACGGACCAGATCGAGATC-3'.

The PCR products were digested with PacI and NotI restriction enzymes and inserted into the $\gamma$ vectors. The specific primers were designed to amplify the target gene. Then the correctness and location of the genome were verified by gene sequencing.

\section{In vitro transcription of viral RNAs and plant inoculation}

The $\alpha, \beta \Delta \beta$, and $\gamma$ plasmids and four modified $\gamma$ plasmids $(\gamma: P D S, \quad \gamma: S B E I I a, \gamma: S S I I a$, and $\gamma: S B E I I a-S S I I a)$ were digested to generate linear plasmids using a monorestriction endonuclease. The linear plasmids were then used for in vitro transcription with the mMessage mMachine T7 using an in vitro transcription kit (Ambion, Austin, TX, USA) according to the manufacturer's recommendations (Ma et al. 2012). The $\alpha, \beta$, and $\gamma$ transcription products were 
mixed in equal amounts to generate BSMV:00. Similarly, the $\alpha, \beta \Delta \beta$, and one of the recombinant $\gamma$ plasmids $(\gamma-P D S$, $\gamma:$ SBEIIa, $\gamma:$ SSIIa, and $\gamma:$ SBEIIa-SSIIa) transcription products were mixed to generate BSMV:PDS, BSMV:SBEIIa, BSMV:SSIIa, and BSMV:SBEIIa-SSIIa, respectively. These mixed infectious viral RNAs were added to inoculation buffer to generate inoculation solutions as described by Scofield et al. (2005).

Wheat spikes were inoculated at heading using the spike-rub method. Briefly, a $20-\mu \mathrm{L}$ aliquot of the inoculation solution was applied onto each spike by gently sliding three pinched fingers from the base to the tip of the spikes five times (Ma et al. 2012). Spikes inoculated with BSMV:00 were used as controls. After inoculation, the spikes were misted with nuclease-free water and then covered with plastic film for $1 \mathrm{~d}$.

\section{Measurement of transcript abundance by qRT-PCR}

To measure the expression levels of target genes, quantitative real-time PCR (qRT-PCR) was performed using DNA Master SYBR Green I chemistry on a Roche LightCycler ${ }^{\circledR} 480$ (Roche Diagnostics, Indianapolis, IN, USA). The gene-specific primers used for qRT-PCR were as follows:

Actin-RT-F: 5'-TGTGCTTGATTCTGGTGATGGTGTG-3', Actin-RT-R: 5'-CGATTTCCCGCTCAGCAGTTGT-3', PDS-RT-F: 5'-TCGAAGGGTTCTATCTGG-3', PDS-RT-R: 5'-CTACAACAATGTGGCAAT-3', SBE II a-RT-F: 5'-GCAGAACTGCGGTCGTGT-3', SBE II $a$-RT-R: 5'-TCCCAGTCATGGCGCTTA -3', SS II $a$-RT-F: 5'-TGCCGCCAAGCTCTACG-3', and SS II $a$-RT-R: 5'-CGTCCGCTCTACTCTGCTAC-3'.

The wheat actin gene (AY423548.1) was used as an internal control for normalization in the VIGS experiments. The following cycling parameters were used: $95^{\circ} \mathrm{C}$ for $2 \mathrm{~min}$, followed by 40 cycles at $95^{\circ} \mathrm{C}$ for $15 \mathrm{~s}, 55^{\circ} \mathrm{C}$ for $30 \mathrm{~s}$, and $72^{\circ} \mathrm{C}$ for $30 \mathrm{~s}$. Relative gene expression was calculated by the $2^{-\Delta \Delta \mathrm{CT}}$ method (Livak and Schmittgen 2001).

\section{Determination of amylose and resistant starch contents}

Spring wheat grains contained approximately $16 \%$ amylose. Grains of BSMV:SBEIIa- or BSMV:SSIIa- or BSMV:SBEIIa-SSIIa-infected wheat spikes at $15 \mathrm{~d}$ post inoculation (dpi) were analyzed using qRT-PCR to detect whether the endogenous target genes were silenced. Target gene-silenced spikes were collected and milled to determine the amylose and resistant starch contents. Amylose content was estimated with an iodometric assay according to the method described by Chrastil (1987), and RS content was determined using the Megazyme Resistant Starch Assay kit (Megazyme Int., Wicklow, Ireland) according to AACC method.

\section{Data analyses}

The means and standard deviations were calculated using Excel 2007 (Microsoft, Redmond, WA, USA) and S.P.S.S. 19.0 (S.P.S.S., Inc., Chicago, IL, USA). Comparisons between groups were conducted using $t$-tests with a significance level of $P<0.01$ or $P<0.05$. Nucleic acid sequences were analyzed using DNAMAN version 5.2.2 (Lynnon Biosoft, San Ramon, CA, USA).

\section{Results}

\section{Establishment of a BSMV-VIGS system for wheat spikes and grains}

To develop an effective BSMV-VIGS system for evaluating gene function in wheat spikes and grains, a test viral vector, $\mathrm{BSMV}: P D S$, was constructed and inoculated on 15 wheat spikes at the heading stage and its effects on photobleaching were assessed. Photobleaching was observed on $86.7 \%$ (13/15) of the BSMV:PDS-inoculated wheat spikes. Photobleaching first appeared at 5-6 dpi, became distinct at 15-16 dpi, and peaked at 25-27 dpi (Fig. 3A). Photobleaching was also observed on grains collected from BSMV:PDS-inoculated spikes at 25 dpi (Fig. 3B). No photobleaching was observed on BSMV:00-inoculated spikes.

We then measured the PDS transcript abundance to confirm that the photo bleaching of spikes and grains was caused by silencing of the endogenous $P D S$ gene. A decrease in $P D S$ transcript abundance was observed in BSMV:PDS-inoculated spikes at 3 dpi. The $P D S$ transcript abundance was the lowest at $15 \mathrm{dpi}$, and PDS gene expression was suppressed until 25 dpi (Fig. 3C). Similarly, the PDS transcript abundance was much lower in grains from BSMV:PDS-inoculated spikes than in grains from BSMV:00-inoculated spikes (Fig. 3D).Collectively, these results suggest that the BSMV-VIGS vector silenced the $P D S$ gene in wheat spikes and grains.

\section{Silencing of the SBEIIa and SSIIa genes separately in wheat grains}

The BSMV-VIGS system was used to evaluate the function of two genes, SBEIIa and SSIIa, involved in the biosynthesis of amylose and RS in wheat grains. To silence SBEIIa or SSIIa, two recombinant BSMV vectors, carrying either a 178-bp SBEIIa fragment (BSMV:SBEIIa) or a 171bp SSIIa fragment (BSMV:SSIIa) were constructed. Then, ten spikes each were inoculated with either BSMV:SBEIIa or BSMV:SSIIa at heading stage. The grains in the middle of the inoculated spikes were collected at $6,9,12,15,18$ and $21 \mathrm{dpi}$ for RNA extraction to examine the changes in the abundance of SBEIIa and SSIIa transcripts through qRTPCR. The SBEIIa transcript abundance decreased approximately $58 \%$ at $6 \mathrm{dpi}, 84 \%$ at $12 \mathrm{dpi}$, and $88 \%$ at 21 


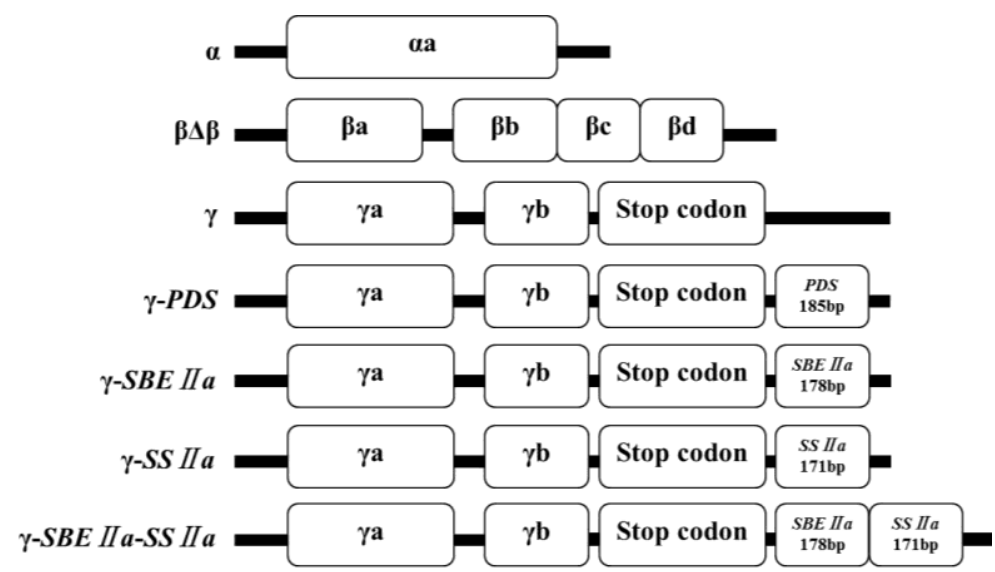

Fig. 1: Genomic organization of BSMV $(\alpha, \beta \Delta \beta$, and $\gamma)$ and the four modified $\gamma$ genomes $(\gamma$-PDS, $\gamma$-SBEIIa, $\gamma$-SSIIa, and $\gamma$-SBEIIa-SSIIa) used in this study. $\alpha, \beta \Delta \beta$, and $\gamma$ are the three parts of the BSMV genome. $\gamma$-PDS, $\gamma$-SBEIIa, $\gamma$-SSIIa, and $\gamma$-SBEIIa-SSIIa are recombinant $\gamma$ vectors carrying cloned fragments of PDS (185 bp), SBEIIa (178 bp), SSIIa (171 bp), and SBEIIa-SSIIa (349 bp), respectively. The SBEIIa-SSIIa fragment (349 bp) is composed of a 178-bp SBEIIa fragment and a 171-bp SSIIa fragment

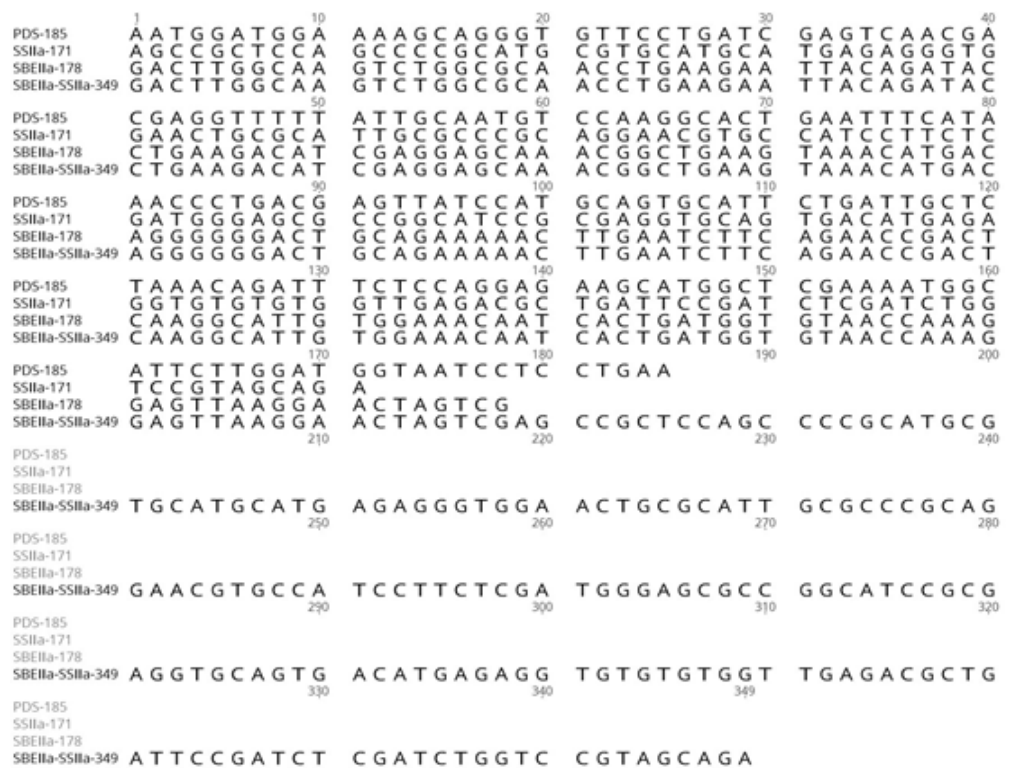

Fig. 2: Gene fragment for recombinant vectors

dpi in the grains of BSMV:SBEIIa-infected spikes compared with the corresponding levels in BSMV:00-inoculated spikes (Fig. 4A). Similarly, the SSIIa transcript abundance decreased approximately $49 \%$ at $6 \mathrm{dpi}, 92 \%$ at $12 \mathrm{dpi}$, and $92 \%$ at 21 dpi in the grains of BSMV:SSIIa-infected spikes compared with the corresponding levels in BSMV:00inoculated spikes (Fig. 4B). These results indicated that the BSMV-VIGS system effectively silenced SBEIIa and SSIIa in wheat grains.

To evaluate the effect of silencing SBE II $a$ and SS II $a$ on the biosynthesis of amylose and RS, ten spikes were inoculated with either BSMV:SBEIIa or BSMV:SSIIa at heading stage. The contents of amylose and RS were determined in mature grains. The average amylose and RS contents of grains from SBEIIa-silenced spikes were 18.62 and $11.61 \%$ higher, respectively, than the contents in control BSMV:00-inoculated spikes (Table 1). Similarly, the amylose and RS contents in SSIIa-silenced spikes were 24.48 and $16.67 \%$ higher, respectively, than those in control BSMV:00-inoculated spikes (Table 1).

\section{Co-silencing of the SBEIIa and SSIIa genes in wheat grains}

After successfully silencing the SBEIIa and SSIIa genes separately, we attempted to explore the usefulness of the BSMV-VIGS system for silencing multiple target genes in wheat grains simultaneously. To co-silence SBEIIa and SSIIa, a BSMV:SBEIIa-SSIIa recombinant vector carrying a 349-bp fragment containing the 178-bp wheat SBEIIa 

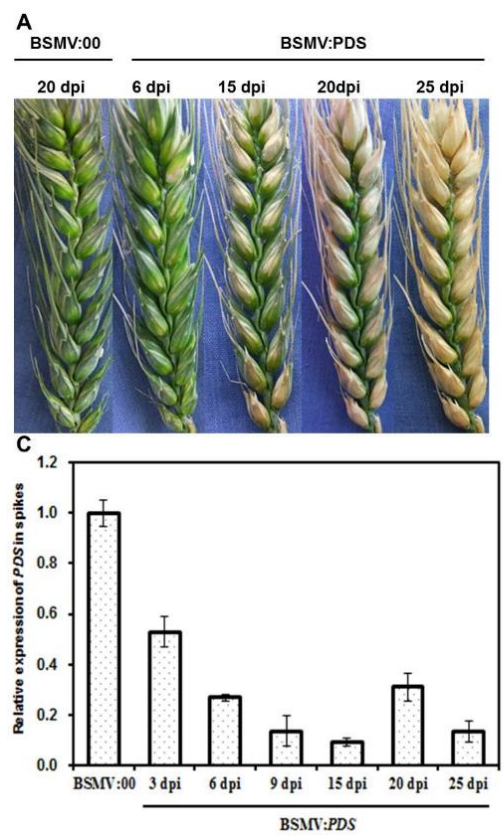

B
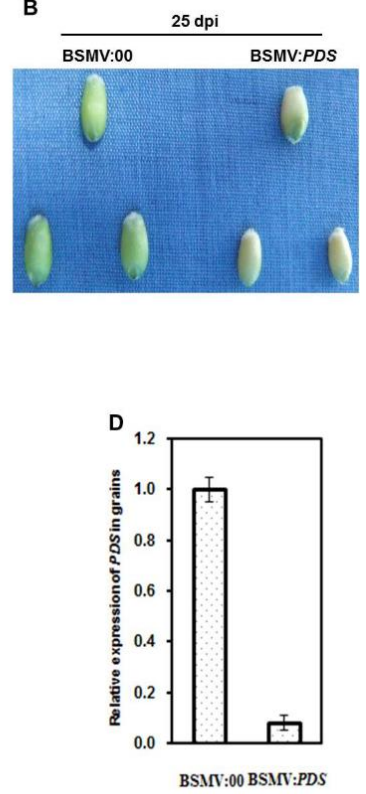

Fig. 3: Silencing of the PDS gene in wheat spikes and grains. A: The far-left spike is a control spike inoculated at heading with BSMV:00 at $20 \mathrm{dpi}$. The next four spikes show the development of photobleaching on a single spike inoculated with BSMV:PDS at heading. The photos were taken at 6, 15, 20, and 25 dpi. B: Grains were collected at 25 dpi from spikes inoculated with BSMV:00 (left) or BSMV:PDS (right). C: Relative PDS expression in wheat spikes inoculated with BSMV:PDS was detected by quantitative real-time PCR (qRT-PCR) at 3, 6, 9, 15, 20, and 25 dpi. D: Relative PDS expression in wheat grains collected at 25 dpi with BSMV:PDS or BSMV:00. Comparisons were made with control spikes inoculated with BSMV:00. Each column represents the mean of three samples; the error bars indicate the standard deviation
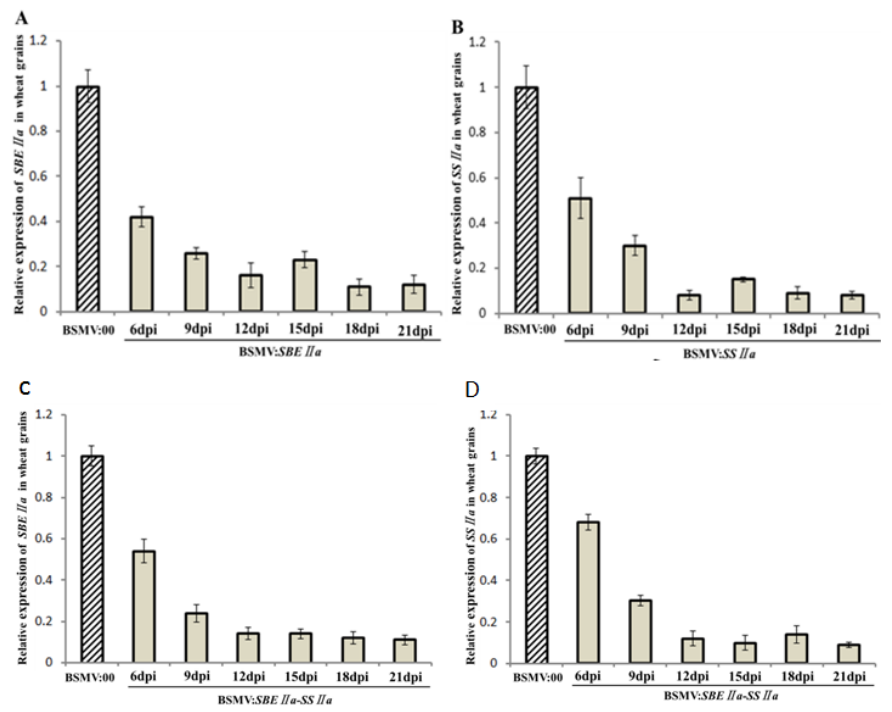

Fig. 4: Relative SBEIIa and SSIIa expression levels in wheat grains. Grains were collected from spikes inoculated with BSMV:SBEIIa, BSMV:SSIIa, and BSMV:SBEIIa-SSIIa at 6, 9, 12, 15, 18, and 21 dpi for RNA isolation. The relative expression levels of SBEIIa (A) and SSIIa (B) in grain samples collected from spikes inoculated with either BSMV:SBEIIa (A) and BSMV:SSIIa (B) were determined by quantitative real-time PCR (qRT-PCR). Relative expression of SBEIIa (C) and SSIIa (D) in grain samples from spikes inoculated with BSMV:SBEIIa-SSIIa was determined by quantitative real-time PCR (qRT-PCR)

fragment and the 171-bp wheat SSIIa fragment was developed. The grains in the middle of the ten BSMV:SBEIIa-SSIIa-inoculated wheat spikes were collected at $6,9,12,15,18$ and 21 dpi for RNA isolation and qRT-PCR. The results showed that the SBEIIa and SSIIa transcripts in grains from BSMV:SBEIIa-SSIIa-inoculated wheat spikes were reduced approximately 46 and $32 \%$ at 6 dpi, 86 and $88 \%$ at $12 \mathrm{dpi}$, and 89 and $90 \%$ at $21 \mathrm{dpi}$, 
Table 1: Average amylose and resistant starch (RS) contents in mature grains from spikes inoculated with BSMV:00, BSMV:SBEIIa, BSMV:SSIIa, and BSMV:SBEIIa-SSIIa

\begin{tabular}{lllc}
\hline Inoculant & Amylose content $(\%)$ & Change in amylosecontent $(\%)$ & RS content $(\%)$ \\
\hline BSMV:00 & $16.75 \pm 0.16$ & & $2.04 \pm 0.02$ \\
BSMV:SBEIIa & $19.87 \pm 0.16^{* *}$ & +18.62 & $2.26 \pm 0.02^{* *}$ \\
BSMV:00 & $16.67 \pm 0.28$ & & $2.04 \pm 0.04$ \\
BSMV:SSIIa & $20.75 \pm 0.23^{* *}$ & +24.48 & $2.38 \pm 0.05 * *$ \\
BSMV:00 & $16.91 \pm 0.14$ & & $2.02 \pm 0.01$ \\
BSMV:SBEIIa-SSIIa & $22.32 \pm 0.37 * *$ & +32.02 & $2.47 \pm 0.03 * *$ \\
\hline
\end{tabular}

Amylose content (\%) and resistant starch (RS) content (\%) are shown as the mean \pm standard deviation. The percent change in amylose content was calculated as follows: amylose content $(\%)=($ amylose content of BSMV:SBEIIa-inoculated spikes - amylose content of BSMV:00-inoculated spikes)/amylose content of BSMV:00-inoculated spikes. The formula used to calculate the change in RS content is the same as that used to calculate the change in amylose content. The average amylose and RS contents in mature grains

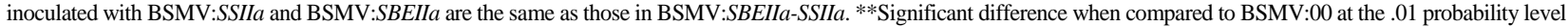

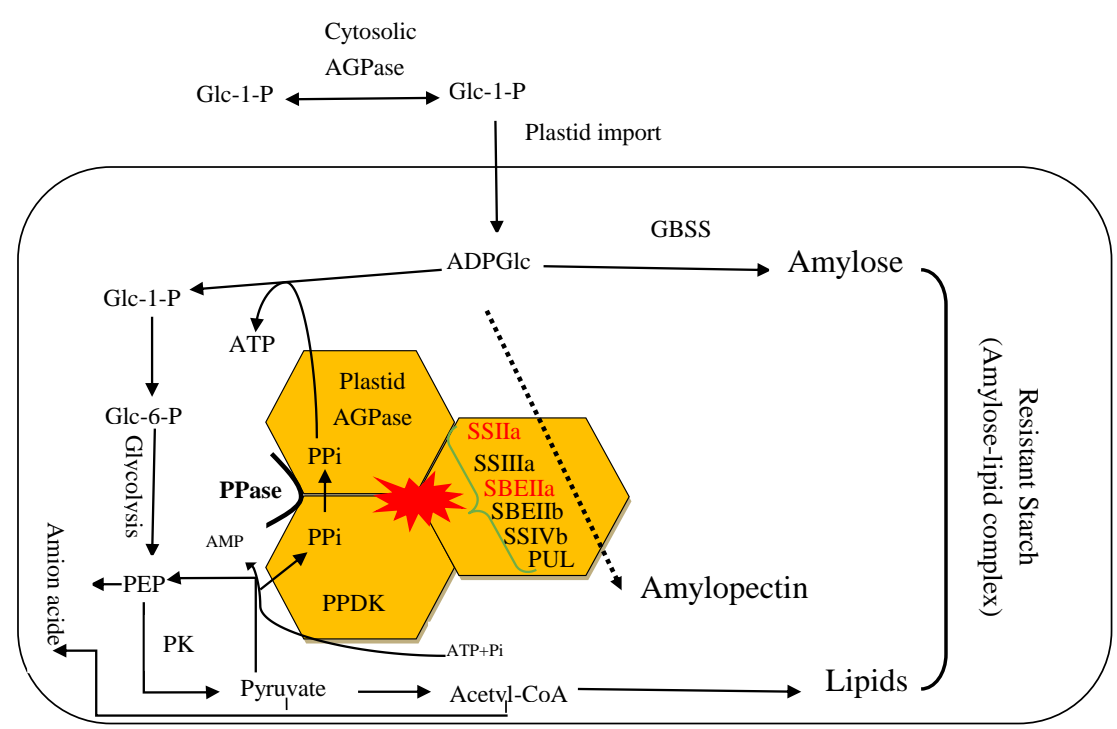

Fig. 5: A proposed starch biosynthesis and metabolic pathway in the plastid. Biosynthesis of ADPGlc is brought about primarily by cytosolic AGPase, and ADPGlc is then imported into the plastid for starch biosynthesis. Amylopectin-synthesizing enzymes, such as SSIIa, SSIIIa, SBEIIa, SBEIIb, SSIVb, and PUL can physically interact with each other to form multienzyme complexes and the complex may also contain other enzymes, such as PPDK and plastid AGPase, that are considered to function in the global regulation of carbon partitioning between starch and lipid. The amyloplast contains a high level of pyrophosphatase, which keeps the concentration of PPi in the stroma very low. PPDK could promote plastid AGPase activity by directly supplying PPi through a substrate-channeling mechanism, resulting in the smooth conversion of ADPGlc to Glc-1-P and enhancing the biosynthesis of lipids. The amylopectin biosynthetic enzymes in the complex are proposed to inhibit the activity of PPDK and AGPase. As a result, ADPGlc is more easily used as a substrate for amylose and amylopectin synthesis by GBSS and amylopectin-synthesizing enzymes, respectively. But when SSIIa and SBEIIa are defective, the interaction between PPDK/AGPase and amylopectin-synthesizing enzymes is disrupted, making AGPase free to channel more ADPGlc for the synthesis of Glc-1-P, a substrate for lipid production. At the same time, the absence of SSIIa and SBEIIa means that relatively more ADPGlc can also be consumed by the Wx protein in the biosynthesis of amylose. Mutation of SSIIa and SBEIIa also causes a defect in amylopectin biosynthesis. This process leads to an increase in amount of amylose-lipid complex or type 5 RS

respectively, compared to the corresponding levels in the grains of BSMV:00-inoculated spikes (Fig. 4C, 4D). These data were similar to those obtained in the experiments in which SBEIIa and SSIIa were silenced separately. Of the ten BSMV:SBEIIa-SSIIa-inoculated spikes, six (60\%) displayed co-silencing of SBEIIa and SSIIa in wheat grains. Two of the remaining inoculated spikes (20\%) only showed silencing of SBEIIa, and the SSIIa transcripts levels in these spikes were the same as those in the control BSMV:00inoculated spikes. These results indicated that the BSMVVIGS system was useful for co-silencing of SBEIIa and SSIIa in wheat grains.

To assess the effects of SBEIIa and SSIIa co-silencing in wheat grains on the biosynthesis of amylose and RS, ten spikes were inoculated with BSMV:SBEIIa-SSIIa at heading stage. Mature grains were collected from BSMV:SBEIIaSSIIa-inoculated spikes to measure the amylose and RS contents. The average amylose contents were $32.03 \%$ higher, and the RS contents were $22.33 \%$ higher in grains from SBEIIa and SSIIa co-silenced spikes than in grains from control BSMV:00-inoculated spikes (Table 1). The amylose content in SBEIIa-silenced grains increased $18.8 \%$ and the $\mathrm{RS}$ content $\mathrm{s}$ increased $10.2 \%$ compared to the corresponding levels in the control (BSMV:00-inoculated; data not shown). These results were similar to those observed in the SBEIIa silencing experiments described above. 


\section{Discussion}

Many traditional approaches that are used to generate mutants, including chemical methods, random mutagenesis, and T-DNA insertions, can be used to evaluate gene function. However, these approaches are technically demanding and inefficient in many plant species. In contrast, VIGS systems are highly efficient and may overcome many of the limitations of the aforementioned methods (Baulcombe 1999; Burch-Smith et al. 2004). Since the BSMV-VIGS system was first successfully applied for the analysis of gene function in barley (Holzberg et al. 2002), it has been widely used in various plants, including wheat (Yang et al. 2020), maize (Murphree et al. 2020), rice (Purkayastha et al. 2010), and Haynaldia villosa (Xing et al. 2018). Studies using the BSMV-VIGS system revealed that three genes, Rarl, Sgt1, and Hsp90, were involved in a powdery mildew resistance pathway containing Mla-13 in barley (Hein et al. 2005).

The BSMV-VIGS system has mainly been used in rapid functional analyses of genes in leaves, although some data on roots and flowers have been published. For example, the BSMV-VIGS system was used to explore the functional genes, powdery mildew resistance genes (Chen et al. 2018), yellow rust resistance genes (Yang et al. 2020), and aphid resistance genes (Kuzuoglu-Ozturk et al. 2012) in leaves. In the present study, two genes related to starch biosynthesis in wheat grains, SBEIIa and SSIIa, were separately or simultaneously silenced using the BSMVVIGS system, which resulted in significant increases in amylose and RS contents. These findings demonstrate that the BSMV-VIGS system is a powerful tool for assessing the functions of genes in wheat grains.

In the first experiment, we used BSMV:PDS to silence the $P D S$ gene in wheat spikes and grains. Photobleaching was observed on the wheat spikes and grains of BSMV:PDSinoculated spikes. qRT-PCR revealed a reduction in $P D S$ transcript levels at $3 \mathrm{dpi}$, which was 2-3 days earlier than when the photobleaching appeared on the wheat spikes. It is possible that photobleaching required a sufficient decrease in $P D S$ transcripts. Differences in photobleaching on the spikes and grains were observed among the BSMV:PDS-inoculated plants; similar differences were detected in a previous study (Ma et al. 2012). The variable efficiency and unstable phenotypes of plants generated using VIGS have been previously described. These variations may not be consistent among different experiments or plants. To solve this problem, the BSMV-VIGS system may need to be optimized for each plant and tissue. In our experiments, we optimized BSMV-VIGS for wheat panicle by comparing different inoculation methods, panicle positions, inoculation times, and culture temperatures. The optimal parameters included inoculation of wheat ears by friction and incubation at $21-$ $24^{\circ} \mathrm{C}$ after inoculation.

Agriculture is considered as the foundation of all food systems and primary source of all the nutrients. Malnutrition and/or disease develop if agriculture cannot supply the nutrient required for good health (Yaseen et al. 2018). Changes in human lifestyle and food consumption have resulted in a large increase in the incidence of type-2 diabetes, obesity, and colon disease, especially in Asia. These conditions are a threat to human health, but consumption of foods high in RS may potentially reduce their incidence. By exploiting natural and induced variation in genes of starch biosynthesis pathways, starch synthesis may be modified to increase the ratio of amylose to amylopectin and other starch properties leading to an increased proportion of resistant starch. Four types of enzymes are required for starch synthesis: starch synthases (SSs) and granule-bound starch synthases (GBSSs), which elongate glucose chains in amylopectin and amylose, respectively, starch branching enzymes (SBEs) that introduce branching points, and starch debranching enzymes (DBEs), which trim branched chains to create a structure that can crystallize to form the granule matrix (Brittany et al. 2020). The SBEIIa and SSIIa genes are closely related to the biosynthesis of amylose and resistant starch. Our results showed that SBEIIa and SSIIa could be silenced using the BSMV-VIGS system. Silencing of SBEIIa and SSIIa increased the amylose and RS contents in wheat grains. This result is in agreement with the findings of studies by Regina et al. (2015) and Sestili et al. (2015). However, the benefit of silencing SSIIa in improving amylose and RS was greater than that of silencing SBEIIa. We postulated that this was probably because silencing SSs would decrease amylopectin biosynthesis and result in a shift in carbon allocation toward amylose biosynthesis through GBSS, which is encoded by the Wx gene and lipid biosynthesis (Zhou et al. 2016).

Based on our findings in this work and published results (Ordonio and Matsuoka 2016; Zhou et al. 2016; Xia et al. 2018), an overview of the main metabolic pathways for starch biosynthesis is shown in Fig. 5. Biosynthesis of ADPGlc is brought about primarily by cytosolic AGPase, and ADPGlc is then imported into the plastid for starch biosynthesis. Amylopectin-synthesizing enzymes, such as SSIIa, SSIIIa, SBEIIa, SBEIIb, SSIVb, and PUL can physically interact with each other to form multienzyme complexes and the complex may also contain other enzymes, such as PPDK and plastid AGPase, that are considered to function in the global regulation of carbon partitioning between starch and lipid. The amyloplast contains a high level of pyrophosphatase, which keeps the concentration of PPi in the stroma very low. And PPDK could promote plastid AGPase activity by directly supplying PPi through a substrate-channeling mechanism, resulting in the smooth conversion of ADPGlc to Glc-1-P and enhancing the biosynthesis of lipids. The amylopectin biosynthetic enzymes in the complex are proposed to inhibit the activity of PPDK and AGPase. As a result, ADPGlc is more easily used as a substrate for amylose and amylopectin synthesis by GBSS and amylopectin-synthesizing enzymes, respectively. But when SSIIa and SBEIIa are defective, the 
interaction between PPDK/AGPase and amylopectinsynthesizing enzymes is disrupted, making AGPase free to channel more ADPGlc for the synthesis of Glc-1-P, a substrate for lipid production. At the same time, the absence of SSIIa and SBEIIa means that relatively more ADPGlc can also be consumed by the Wx protein in the biosynthesis of amylose. Mutation of SSIIa and SBEIIa also causes a defect in amylopectin biosynthesis. This process leads to an increase in amount of amylose-lipid complex or type $5 \mathrm{RS}$. In our study the benefit of silencing SS IIa and SBE II in improving amylose was greater than that of resistant starch. According to published result (Zhou et al. 2016), we speculated that this may be due to the low expression of $\mathrm{Wx}$ gene, and result in more carbon allocation toward lipid biosynthesis.

\section{Conclusion}

We showed that the BSMV-VIGS system is a powerful tool for assessing gene functions in wheat grains. We further demonstrated that the BSMV-VIGS system may be used to silence SBEIIa and SSIIa in wheat grains separately and simultaneously. The benefit of silencing SSIIa in improving amylose and RS was greater than that of silencing SBEIIa. Our results provide fundamental information for improving $\mathrm{RS}$ contents in wheat as well as other cereal crops for improved health benefits among the global population. In the future, high-amylose and high-RS wheat may be produced through the targeted mutagenesis of SSIIa by CRISPR/Cas9 or/and other breeding methods.

\section{Acknowledgments}

We sincerely acknowledge Dr. Meng Ma (College of Life Sciences, Northwest A \& F University) for thoughtful discussions and advice on BSMV inoculation. We acknowledge Zhenxiang Lu from Lethbridge Research Centre, Agriculture and Agri-Food, Canada for providing the VIGS plasmid. This work was supported by the Natural Science Foundation of China (Grant nos. 31160279 and 31260357).

\section{Author Contributions}

Zhaofeng Li performed the concepts, design, definition of intellectual content, literature search, data acquisition, data analysis, and manuscript preparation. Weihua $\mathrm{Li}$ and Wei Liu provided assistance for data acquisition, data analysis, and statistical analysis. Fubo Nan and Donghai Zhang reviewed the manuscript. All authors have read and approved the content of the manuscript.

\section{Conflict of Interest}

The autors declare that they have no conflict of interest.

\section{Ethics Approval}

Not applicable.

\section{Data Availability}

The data obtained in this study is available from the corresponding author upon reasonable request.

\section{References}

Asp NG, I Björck, M Nyman (1993). Physiological effects of cereal dietary fibre. Carbohydr Polymers 21:183-187

Bao YF, ZY Wang, YM Gao, HH Yang, H Zhang, JB Jiang, JF Li, TT Zhao, XY Xu (2020). Silencing of SLB3 transcription factor reduces salt tolerance in tomato. Intl J Agric Biol 24:479-485

Baulcombe DC (1999). Fast forward genetics based on virus-induced gene silencing. Curr Opin Plant Biol 2:109-113

Bennypaul HS, Mutti JS, Rustgi S, Kumar N, Okubara PA, Gill KS (2012). Virus-induced gene silencing (VIGS) of genes expressed in root, leaf, and meiotic tissues of wheat. Funct Integr Genomics $12: 143-156$

Brittany H, K Trafford, A Lovegrove, S Griffiths, C Uauy, P Shewry (2020). Strategies to improve wheat for human health. Nat Food 1:475-480

Brown IL (2004). Applications and uses of resistant starch. J AOAC Intl $87: 727-732$

Burch-Smith TM, JC Anderson, GB Martin, SP Dinesh-Kumar (2004). Applications and advantages of virus-induced gene silencing for gene function studies in plants. Plant J 39:734-746

Calvo-Baltanás V, CL Wijnen, C Yang, N Lukhovitskaya, CBD Snoo, L Hohenwarter, JJB Keurentjes, HD Jong, A Schnittger, E Wijnker (2020). Meiotic crossover reduction by virus-induced gene silencing enables the efficient generation of chromosome substitution lines and reverse breeding in Arabidopsis thaliana. Plant J 104:1437-1452

Chen G, B Wei, G Li, C Gong, R Fan, X Zhang (2018). TaEDS1 genes positively regulate resistance to powdery mildew in wheat plant. Mol Biol 96:607-625

Chrastil J (1987). Improved colorimetric determination of amylose in starches or flours. Carbohydr Res 159:154-158

Cox DE, S Dyer, R Weir, X Cheseto, M Sturrock, D Coyne, B Torto, AG Maule, JJ Dalzell (2019). ABC transporter genes $A B C$ - $C 6$ and $A B C$ $G 33$ alter plant-microbe-parasite interactions in the rhizosphere. $S c i$ Rep 9; Article 19899

Eck LV, T Schultz, JE Leach, SR Scofield, FB Peairs, AM Botha, NL Lapitan (2010). Virus-induced gene silencing of WRKY53 and an inducible phenylalanine ammonia-lyase in wheat reduces aphid resistance. Plant Biotechnol J 8:1023-1032

Gunupuru LR, A Perochon, SS Ali, SR Scofield, FM Doohan (2019). Virus-induced gene silencing (VIGS) for functional characterization of disease resistance genes in barley seedlings. In: Barley, Vol. 1900, pp:95-114. Harwood W (Ed.). Humana Press, New York, New York, USA

Hazard B, X Zhang, P Colasuonno, C Uauy, DM Beckles, J Dubcovsky (2012). Induced mutations in the starch branching enzyme II (SBEII) genes increase amylose and resistant starch content in durum wheat. Crop Sci 52:1754-1766

Hein I, M Barciszewska-Pacak, K Hrubikova, S Williamson, M Dinesen, IESoenderby, S Suresh, A Jarmolowski, K Shirasu, C Lacomme (2005). Virus-induced gene-silencing based functional characterization of genes associated with powdery mildew resistance in barley. Plant Physiol 138:2155-2164

Holzberg S, P Brosio, C Gross, GP Pogue (2002). Barley stripe mosaic virus-induced gene silencing in a monocot plant. Plant J 30:315-327

Hylla S, A Gostner, G Dusel, H Anger, HP Bartram, SU Christl, H Kasper, W Scheppach (1998). Effects of resistant starch on the colon in healthy volunteers: Possible implications for cancer prevention. Amer J Clin Nutr 67:136-142 


\section{VIGS of SBEIIa and SSIIa in Triticum aestivum / Intl J Agric Biol, Vol 25, No 6, 2021}

Kim WK, MK Chung, NE Kang, MH Kim, OJ Park (2003). Effect of resistant starch from corn or rice on glucose control, colonic events, and blood lipid concentrations in streptozotocin-induced diabetic rats. J Nutr Biochem 14:166-172

Kuzuoglu-Ozturk D, O Cebeci Yalcinkaya, BA Akpinar, G Mitou, G Korkmaz, D Gozuacik, H Budak (2012). Autophagy-related gene, TdAtg8, in wild emmer wheat plays a role in drought and osmotic stress response. Planta 236:1081-1092

Li J, G Jiao, Y Sun, J Chen, Y Zhong, L Yan, D Jiang, Y Ma, L Xia (2020). Modification of starch composition, structure and properties through editing of TaSBEIIa in both winter and spring wheat varieties by CRISPR/Cas9. Plant Biotechnol J 25:1-15

Livak KJ, TD Schmittgen (2001). Analysis of relative gene expression data using real-time quantitative PCR and the 2(-Delta Delta C(T)) Method. Methods 25:402-408

Lopez HW, MA Levrat-Verny, C Coudray, C Besson, V Krespine, A Messager, C Demigne, C Remesy (2001). Class 2 resistant starches lower plasma and liver lipids and improve mineral retention in rats. $J$ Nut 131:1283-1289

Ma M, Y Yan, L Huang, M Chen, H Zhao (2012). Virus-induced genesilencing in wheat spikes and grains and its application in functional analysis of HMW-GS-encoding genes. BMC Plant Biol 12; Article 141

Moreau NM, LJ Martin, CS Toquet, CL Laboisse, PG Nguyen, BS Siliart, HJ Dumon, MM Champ (2003). Restoration of the integrity of rat caeco-colonic mucosa by resistant starch, but not by fructooligosaccharides, in dextran sulfate sodium-induced experimental colitis. Brit J Nutr 90:75-85

Morell MK, B Kosar-Hashemi, M Cmiel, MS Samuel, P Chandler, S Rahman, A Buleon, IL Batey, Z Li (2003). Barley sex6 mutants lack starch synthase IIa activity and contain a starch with novel properties. Plant J 34:173-185

Murphree C, SB Kim, S Karre, R Samira, P Balint-Kurti (2020). Use of virus-induced gene silencing to characterize genes involved in modulating hypersensitive cell death in maize. Mol Plant Pathol 21:1662-1676

Ordonio RL, M Matsuoka (2016). Increasing resistant starch content in rice for better consumer health. Proc Nat Acad Sci USA 113:12616-12618

Purkayastha A, S Mathur, V Verma, S Sharma, I Dasgupta (2010). Virusinduced gene silencing in rice using a vector derived from a DNA virus. Planta 232:1531-1540

Rana TM (2007). Illuminating the silence: Understanding the structure and function of small RNAs. Nat Rev Mol Cell Biol 8:23-36

Ratcliff F, BD Harrison, DC Baulcombe (1997). A similarity between viral defense and gene silencing in plants. Science 276:1558-1560

Regina A, P Berbezy, B Kosar-Hashemi, S Li, M Cmiel, AR Larroque O Bird, SM Swain, C Cavanagh, SA Jobling, Z Li, M Morell (2015). A genetic strategy generating wheat with very high amylose content Plant Biotechnol J 13:1276-1286

Regina A, A Bird, D Topping, S Bowden, J Freeman, T Barsby, B KosarHashemi, Z Li, S Rahman, M Morell (2006). High-amylose wheat generated by RNA interference improves indices of large-bowel health in rats. Proc Nat Acad Sci USA 103:3546-3551

Scofield SR, Huang L, Brandt AS, Gill BS (2005). Development of a virusinduced gene-silencing system for hexaploid wheat and its use in functional analysis of the Lr21-mediated leaf rust resistance pathway. Plant Physiol 138:2165-2173
Sestili F, S Palombieri, E Botticella, P Mantovani, R Bovina, D Lafiandra (2015). TILLING mutants of durum wheat result in a high amylose phenotype and provide information on alternative splicing mechanisms. Plant Sci 233:127-133

Sestili F, M Janni, A Doherty, E Botticella, R D'Ovidio, S Masci, HD Jones, D Lafiandra (2010). Increasing the amylose content of durum wheat through silencing of the SBEIIa genes. BMC Plant Biol 10; Article 144

Tang J, D Yang, J Wu, S Chen, L Wang (2020). Silencing JA hydroxylases in Nicotiana attenuate enhances jasmonic acid-isoleucine-mediated defenses against Spodoptera litura. Plant Divers 42:111-119

Topping DL, PM Clifton (2001). Short-chain fatty acids and human colonic function: Roles of resistant starch and nonstarch polysaccharides. Physiol Rev 81:1031-1064

Umemoto T, M Yano, H Satoh, A Shomura, Y Nakamura (2002). Mapping of a gene responsible for the difference in amylopectin structure between japonica-type and indica-type rice varieties. Theor Appl Genet 104:1-8

Waterhouse PM, MB Wang, T Lough (2001). Gene silencing as an adaptive defence against viruses. Nature 411:834-842

Xia J, D Zhu, RM Wang, Y Cui, YM Yan (2018). Crop resistant starch and genetic improvement: A review of recent advances. Theor Appl Genet 131:2495-2511

Xing L, P Hu, J Liu, K Witek, S Zhou, J Xu, W Zhou, L Gao, Z Huang, R Zhang, X Wang, P Chen, H Wang, JDG Jones, M Karafiátová, J Vrána, J Bartoš, J Doležel, Y Tian, Y Wu, A Cao (2018). Pm21 from Haynaldia Villosa encodes a CC-NBS-LRR protein conferring powdery mildew resistance in wheat. Mol Plant 11:874-878

Yamamori M, S Fujita, K Hayakawa, J Matsuki, T Yasui (2000). Genetic elimination of a starch granule protein, SGP-1, of wheat generates an altered starch with apparent high amylose. Theor Appl Genet 101:21-29

Yan JK, NN Zhang, XL Wang, SQ Zhang (2018). Selection of yield-related traits for wheat breeding in semi-arid region. Intl J Agric Biol 20:569-574

Yang Q, MA Islam, K Cai, S Tian, Y Liu, Z Kang, J Guo (2020). TaClpS1, negatively regulates wheat resistance against Puccinia striiformis $\mathrm{f}$. spp. tritici. BMC Plant Biol 20; Article 555

Yaseen M, T Abbas, MZ Aziz, A Wakeel, H Yasmeen, W Ahmed, A Ullah, M Naveed (2018). Microbial assisted foliar feeding of micronutrients enhance growth, yield and biofortification of wheat. Intl J Agric Biol 20:353-360

Young GP, RKL Leu (2004). Resistant starch and colorectal neoplasia. $J$ AOAC Intl 87:775-786

Yousaf S, G Rasool, I Amin, S Mansoor, M Saeed (2013). Interference of a synthetic rep protein to develop resistance against cotton leaf curl disease. Intl J Agric Biol 15:1140-1144

Zhang X, C Colleoni, V Ratushna, M Sirghie-Colleoni, M James, A Myers (2004). Molecular characterization demonstrates that the Zea mays gene sugary2 codes for the starch synthase isoform SSIIa. Plant Mol Biol 54:865-879

Zhou H, L Wang, G Liu, X Meng, Y Jing, X Shu, X Kong, J Sun, H Yu, SM Smith, D Wu, J Li (2016). Critical roles of soluble starch synthase SSIIIa and granule-bound starch synthase waxy in synthesizing resistant starch in rice. Proc Nat Acad Sci USA 113:12844-12849

Zhou J, RJ Martin, RT Tulley, AM Raggio, L Shen, E Lissy, K McCutcheon, MJ Keenan (2009). Failure to ferment dietary resistant starch in specific mouse models of obesity results in no body fat loss. J Agric Food Chem 57:8844-885 